Working Paper 95-59

Economics Series 29

December 1995
Departamento de Economía Universidad Carlos III de Madrid Calle Madrid, 126 28903 Getafe (Spain)

Fax (341) 624-9875

\title{
DIFFERENTIAL-DIFFERENCE EQUATIONS IN ECONOMICS: ON THE NUMERICAL SOLUTION OF VINTAGE CAPITAL GROWTH MODELS
}

\author{
Raouf Boucekkine, Omar Licandro and Christopher Paul*
}

\begin{abstract}
In this paper, we examine techniques for the analytical and numerical solution of statedependent differential-difference equations. Such equations occur in the continuous time modelling of vintage capital growth models, which form a particularly important class of models in modern economic growth theory. The theoretical treatment of non-statedependent differential-difference equations in economics has already been discussed by Benhabib and Rustichini (1991). In general, though, the state-dependence of a model prevents its analytical solution in all but the simplest of cases. We review a numerical method for solving state-dependent models, using some simple examples to illustrate our discussion. In addition, we analyse the Solow vintage capital growth model. We conclude by mentioning a crucial unresolved issue related to this topic.
\end{abstract}

Key Words

Economic growth theory; Vintage capital; Differential-difference equations; State-dependence; Numerical solution

* Raouf Boucekkine, Universidad Carlos III de Madrid. Omar Licandro, FEDEA and Universidad Carlos III de Madrid. Christopher Paul, Mathematics Department, Manchester University. 



\section{Introduction}

The modelling of time heterogeneity, such as the simultaneous presence of goods and assets of different ages, remains one of the most difficult issues in both theoretical and empirical economics. Clearly a treatment of time heterogeneity is desirable in order to improve the realism of models, as most real-world economic systems involve such heterogeneity. Moreover, in certain elementary cases, diversifying the time structure of goods and assets can become a key economic decision. To illustrate this point, we address the issue of capital replacement decisions, a topic developed by Solow et al (1966): In a growing economy, due to exogeneous technological progress, a key task is to ensure that old machines are efficiently replaced by new machines. In this type of model, referred to as a vintage capital growth model, the replacement decisions consist of determining the optimal age structure for the productive capital, since technological progress is continuously incorporated into new machines. In short, the replacement decisions correspond to determining the optimal time heterogeneity of the machines currently in use.

The original continuous time modelling of this problem included a number of technical difficulties that led to the decline of this approach in the late seventies and early eighties. (See Malcomson (1975) for a discussion of the difficulties ${ }^{1}$.) However, at the beginning of this decade, research on this class of models was resumed. This was due to the fact that observations of many national economies revealed clear inconsistencies with the standard neoclassical homogeneous capital growth model. A detailed analysis of these inconsistencies can be found in Cooley et al (1994). These inconsistencies motivated a number of papers aimed at modelling and analyzing the replacement decisions problem mentioned above. This paper is intended to be a technical contribution to this discussion.

One way to overcome the technical difficulties that arise in the analysis of the optimal replacement strategy is simply to exogenize the latter problem, as in the case of Benhabib and Rustichini (1991) who consider some typical replacement rules within a partial equilibrium setting. Of course, in such frameworks, the replacement decisions are no longer endogenous as they should be, but this approach represents a worthwhile step towards a complete treatment of the problem. Moreover, it provides the first insight into the richer dynamics of vintage capital growth models compared to those of the standard neoclassical model. More precisely, the exogeneous replacement rules used by Benhabib and Rustichini give rise to differential-difference equations with a constant lag (or span) that can be solved using the Laplace transform approach of Bellman and Cooke (1963).

\footnotetext{
${ }^{1}$ Some of the difficulties have recently been solved by Van Hilten (1991).
} 
In this paper, we argue that the replacement decisions problem can be completely analyzed, at least numerically, using general differential-difference equations with flexible endogenous lags. We show that the vintage capital growth model of Solow et al (1966) (hereafter SVCM) can be transformed into a system of differential-difference equations of the form

$$
y^{\prime}(t)=F[t, y(t), y(t-\tau(t, y(t)))]
$$

where $t$ is the time index, $y(t)$ is the vector of endogenous variables, $F(\cdot)$ is an appropriately dimensioned vector function, and $\tau(\cdot)$ is a real-valued function. In the case studied here, $\tau(\cdot)$ is positive and corresponds to the lag. The resulting differential system is called a delay differential equation (DDE) system. Note that in equation (1), the lag is not only timedependent, it is state-dependent in the sense that it depends on the endogenous variables $y(t)$. Indeed, when the replacement decisions are endogenous, as in the $\mathrm{SVCM}^{2}$, the lags are strongly related to the endogenous replacement decisions for some elementary reasons that we will indicate later. Unfortunately, the Laplace transform approach is not very useful either for solving or for investigating the stability properties of non-constant lag DDEs.

Indeed, any analytical solution seems impossible in the case of a general state-dependent DDE. Even calculating the numerical solution is far from being trivial. In this paper, we use some recent developments in computational mathematics to provide a basic numerical framework for solving models in the form of equation $(1)^{3}$. The numerical code that we use is based on the work of Baker and Paul (1993-a, 1993-b). The numerical methods for DDEs are an obvious extension of the methods for solving ordinary differential equations (ODEs), namely linear multistep and Runge-Kutta methods, except that state-dependent lags may require special numerical treatment.

This paper is organized as follows: In Section 2 we state more precisely the numerical problem that we address. Section 3 reviews the main principles underlying the solution techniques needed to solve state-dependent DDEs, using simple examples to illustrate the arguments. Section 4 presents an application of these numerical techniques to an extended version of the SVCM. Finally, we conclude by mentioning a crucial unresolved numerical issue that we hope will open a new and promising area of computational economics.

\footnotetext{
${ }^{2}$ For a more recent contribution, see Caballero and Hammour (1994).

${ }^{3}$ The solution technique can also be applied to neutral equations, where the differential-difference equations depend on delayed derivative terms.
} 


\section{Basic Concepts and Solution Methods}

In this section, we introduce some techniques for solving DDEs, starting with the constant lag case. Consider the following scalar constant lag DDE:

$$
y^{\prime}(t)=y(t-\tau) \quad t \geq 0
$$

where $\tau>0$. Note that the essential difference between ODEs and DDEs is that the usual initial condition $y(0)$ required by an ODE is no longer sufficient to be able to solve a DDE, such as equation (2). Instead we need an initial function $y_{0}(t)$ specified over the interval $[-\tau, 0]$ to be able to solve equation (2). Given a continuous initial function, it is easy to find an analytical expression for the continuous solution of (2) using elementary integration. For example, if $y_{0}(t)=a$ where $a$ is a constant, then $y^{\prime}(t)=a$ on $[0, \tau]$ yielding $y(t)=(t+1) a$ on this interval, since $y(0)=y_{0}(0)=a$. We can extend the range of the solution in this way for as long as we please, on any interval of the form $[k \tau,(k+1) \tau]$ for integer $k>0$. This technique is called the method of steps in computational mathematics. The points $\{0, \tau, 2 \tau, \ldots\}$ are called the meshpoints associated with the method of steps.

We now apply the method of steps to a more general equation that cannot be solved analytically. The method of solving the following example clearly demonstrates the connection between ODE and DDE solvers, a connection that we will exploit later. Consider the scalar equation

$$
y^{\prime}(t)=G[y(t), y(t-\tau)] \quad t \geq 0
$$

where both $\tau>0$ and $y(t)=y_{0}(t)$ on $[-\tau, 0]$ are given, and $G(\cdot)$ is any real-valued function. Unlike equation (2), it is obvious that in general we cannot solve equation (3) analytically for $y(t)$ on the interval $[0, \tau]$. To do so would require the integration of the function $G\left[y(t), y_{0}(t-\tau)\right]$, or equivalently the solution of the ODE

$$
y^{\prime}(t)=G\left[y(t), y_{0}(t-\tau)\right]
$$

which typically does not have an analytical solution. Therefore an ODE solver is used to solve equation (3) numerically on the interval $[0, \tau]$, and this approach results in the following method of steps algorithm (MSA). The MSA can be described as follows:

1. Given $y_{0}(t)$, determine $y(t)$ on the interval $[0, \tau]$ by solving the ODE

$$
y^{\prime}(t)=G\left[y(t), y_{0}(t-\tau)\right]
$$

Denote the solution of this ODE by $y_{1}(t)$. 
2. For each $k \geq 2$, given $y_{k-1}(t)$, determine $y(t)$ on the interval $[(k-1) \tau, k \tau]$ by solving the ODE

$$
y^{\prime}(t)=G\left[y(t), y_{k-1}(t-\tau)\right] .
$$

Denote the solution of this ODE by $y_{k}(t)$.

Thus the solution of equation (3) is given by $y_{k}(t)$ for $t \in[(k-1) \tau, k \tau]$ for integer $k \geq 1$.

REMARK 1: Particular attention should be paid to the connection between ODE and DDE solvers. At each step of the MSA, we use an ODE solver to obtain the numerical solution. There are two main classes of numerical method for solving ODEs: Runge-Kutta methods and linear multistep methods. Both of them are based on quadrature techniques, and usually only produce a discrete solution, so that computing the "continuous time" solution requires interpolation. ODE solvers are now included in a wide variety of software packages (for example, MATLAB), and as such this part of the algorithm can be easily implemented. REMARK 2: The MSA can be extended to systems of DDEs without any difficulty, since solving systems of ODEs does not involve any further theoretical or practical difficulties. However, it is unlikely that all the variables in the system of DDEs are delayed ${ }^{4}$. For a non-delayed variable $y^{\mathrm{M}}(t)$, say, according to ODE theory, only an initial condition $y^{\mathrm{M}}(0)$ is needed in order to be able to calculate the solution.

REMARK 3: Another important issue is the propagation of derivative discontinuities in the solution. A quick look at the solution $y_{1}(t)$ of equation (2) at the first meshpoint $t=0$ is sufficient to demonstrate one of the most significant features of DDEs: Namely that some derivatives of the solution are discontinuous at the meshpoints. These derivative discontinuities can propagate forward in time in the solution, the exact propagation being dependent on the system of DDEs. Fortunately this issue is not the most complicated one in the solution of DDEs, as there already exists sufficient theory allowing the problem of derivative discontinuities to be addressed quite easily. (See, for example, Willé and Baker (1992).)

REMARK 4: In many economic applications, the system of DDEs is obtained by differentiating the structural equations of the model valid for, say, $t \geq 0$ and from the initial functions valid for $t<0$. In the specification of the model, $y(0)$ is not usually given explicitly (unlike in equations (2) and (3)), although it can be determined from the original system of structural equations by putting $t=0$. Thus, before solving the resulting system of DDEs, we have to compute $y(0)$ from the structural equations. This is always possible

\footnotetext{
${ }^{4}$ See, for example, our economic application in Section 4.
} 
as the models yielding DDEs are typically backward-looking. Hereafter we assume that $y(0)$ has already been calculated from the structural equations.

Note that even if an analytical solution could be obtained using the MSA, it would not be particularly useful for studying the stability of the DDE. However the Laplace transform approach developed by Bellman and Cooke (1963), in which solutions are expressed as sums of exponential terms, does permit a better investigation of the stability. Benhabib and Rustichini (1991) applied this approach to vintage capital growth models with exogenous replacement rules. However, as we have already stated, this approach is only possible when the lags are constant, which means that it is not applicable to state-dependent DDEs.

In fact, even the MSA as introduced earlier has problems when a lag is either timevarying state-independent or state-dependent. To illustrate this point, consider a timevarying state-independent $\mathrm{DDE}^{5}$

$$
y^{\prime}(t)=y(-\sin (t)) \quad t \geq 0
$$

where $y_{0}(t)=1$ for $t \leq 0$. The lag function is $\tau(t)=t+\sin (t)$, and is clearly time-varying. We cannot apply the MSA to solve the DDE immediately, because the meshpoints are not directly available as before. If the lag was state-dependent, then there would be no way to determine the meshpoints of the MSA without solving the DDE first! ${ }^{6}$

We address the question of the availability of the meshpoints when DDEs are timevarying and/or state-dependent in the next section. We modify the MSA in such a way that we avoid the latter difficulty, taking advantage of recent developments related to this topic.

\section{Numerical Solution}

In this section, we address the problem of the unavailability of meshpoints. We motivate our discussion using two simple examples to illustrate the arguments. More theoretical details can be found in Baker and Paul (1993-a, 1993-b).

\footnotetext{
${ }^{5}$ Note that the interval on which the initial function $y_{0}(t)$ must be defined, in order to allow the DDE to be solved, is $\left[\min _{t \geq 0}\{t-\tau(\cdot)\}, 0\right]$. Thus for a general time-varying state-independent DDE the length of this interval may not be immediately obvious, and in the case of a state-dependent DDE it cannot be determined until the solution is known!

${ }^{6} \mathrm{~A}$ less immediate barrier to the application of the MSA is the possibility of a vanishing lag. i.e., When the lag $\tau(t)$ tends to zero for some admissible value of $t, t^{\sharp}$ say. In this case, the lengths of the solution intervals in the MSA also tend to zero, so that the MSA fails to progress beyond $t^{\sharp}$. This problem has recently been addressed in the case of continuous explicit Runge-Kutta methods by Baker and Paul (1993-a), who use a predictor-corrector approach.
} 
The only way to solve the problem of the unavailability of meshpoints is to decompose each step of the MSA as follows:

1. Given the initial function, solve the DDE on an unknown interval.

2. Determine the interval on which the computed solution is valid.

In the original MSA, given the meshpoints, we solved the DDE interval by interval. Here, this approach is reversed.

We can apply this strategy, for example, to equation (4): Given that $y_{0}(t)=1$ for $t \leq 0$, it follows that $y(t)=t+1$ on an unknown interval $\left[\sigma_{0}=0, \sigma_{1}\right]$. To compute the unknown meshpoint $\sigma_{1}$, we have to find the interval $\left[0, \sigma_{1}\right]$ on which the computed solution $y(t)=t+1$ is valid. This can be done quite naturally by analogy with the constant lag case (2). In the latter case, the meshpoint $\sigma_{k+1}$ is obtained from the meshpoint $\sigma_{k}$ by adding $\tau$ to $\sigma_{k}$. This strategy (used in the MSA) yields the value of $\sigma_{1}$ by solving

$$
\sigma_{1}-\tau\left(\sigma_{1}\right)=\sigma_{0}=0
$$

which for equation (4) means solving $-\sin \left(\sigma_{1}\right)=0$. We have chosen this example to illustrate a further difficulty, namely that there can be a problem with non-uniqueness of values for the meshpoint. In the case of equation (4), $\sigma_{1}=k \pi$ for any non-zero integer $k$. However the non-uniqueness problem is quite easily solved. If $\sigma_{1}=2 \pi$ then $y(t)=t+1$ on $[0,2 \pi]$, but $-\sin (t)>0$ for $t \in[\pi, 2 \pi]$ so that $y^{\prime}(t)=y(-\sin (t))=-\sin (t)+1$. Thus $y(t)=\cos (t)+t+2$ on $[\pi, 2 \pi]$, which leads to a contradiction. The only value of $\sigma_{1}$ that does not lead to a contradiction is $\sigma_{1}=\pi$, so the correct meshpoint value is the smallest one. This is quite a general result in practice, and usually only the smallest meshpoint value is the correct one. It is possible that in some degenerate cases, that we do not discuss here, a larger meshpoint value is more convenient, but this does not present any problem as the selection criterion is clear: Pick the largest meshpoint value $\sigma$, say, such that the derivative of the solution is consistent with the DDE over the whole interval $[0, \sigma]$. Thus the non-uniqueness problem is relatively easy to solve when it does arise.

The same approach can be used to solve state-dependent DDEs. In this case the lag functions can depend on solution values from the current interval ${ }^{7}$, thus the length of the current interval may depend on the solution for the current interval. Therefore we need to solve the state-dependent DDE before we can determine the meshpoint. To illustrate this point, consider the simple state-dependent DDE

$$
y^{\prime}(t)=y(y(t)), \quad y_{0}(t)=2, \quad y(0)=-1,
$$

\footnotetext{
${ }^{7} \mathrm{By}$ "current interval", we mean the interval that is currently being solved.
} 
where the lag $\tau(t, y(t))=t-y(t)$. As before, we compute the solution $y(t)$ on the interval $\left[\sigma_{0}=0, \sigma_{1}\right]$ and then calculate $\sigma_{1}$. Substituting the initial function into the DDE, on the first interval $\left[0, \sigma_{1}\right]$ we have that $y^{\prime}(0)=y(-1)=2$ and obtain the ODE

$$
y^{\prime}(t)=2,
$$

so that $y(t)=2 t-1$ on $\left[0, \sigma_{1}\right]$. Having obtained $y(t)$, it remains to determine the interval on which the solution is valid. Using the same strategy as before, we compute the value of $\sigma_{1}$ by solving

$$
\sigma_{1}-\tau\left(\sigma_{1}, y\left(\sigma_{1}\right)\right)=\sigma_{0}=0
$$

ensuring that we use the correct expression for the solution in $\tau(\cdot)$. Substituting the computed solution $y(t)=2 t-1$ into $\tau(\cdot)$, we get

$$
\left(2 \sigma_{1}-1\right)=0
$$

so that $\sigma_{1}=\frac{1}{2}$. Hence, for equation (5), $y(t)=2 t-1$ for $t \in\left[0, \frac{1}{2}\right]$. We can repeat this process at each stage of the MSA, allowing the range of the solution to be extended indefinitely.

Thus the only additional difficulty involved in solving DDEs with time-varying and/or state-dependent lags is the computation of the meshpoints. The most general DDE that we consider here can be expressed in the form of equation (1),

$$
y^{\prime}(t)=F[t, y(t), y(t-\tau(t, y(t)))]
$$

Computing the meshpoint $\sigma_{k+1}$, given the previously computed solution $y(t)=y_{i+1}(t)$ on $\left[\sigma_{i}, \sigma_{i+1}\right]$ for $i=0, \ldots, k$, involves solving the equation

$$
\sigma_{k+1}-\tau\left(\sigma_{k+1}, y\left(\sigma_{k+1}\right)\right)=\sigma_{i}
$$

for the meshpoint $\sigma_{i}$ that yields the smallest value for $\sigma_{k+1}>\sigma_{k}$. This is clearly a nontrivial task due to the possible non-linearity of both $\tau(\cdot)$ and $y(t)$. Further difficulties can arise from the fact that, in general, the solution can only be computed numerically. In practice, for the DDEs that arise in economic modelling, the non-uniqueness of meshpoints problem is less complicated than suggested by equation (6), because the lag functions used are generally monotonic ${ }^{8}$.

However, since numerical methods for solving DDEs involve a number of numerical tools (quadrature-based techniques for solving the ODEs, interpolation methods for providing

\footnotetext{
${ }^{8}$ The lag functions are linear with respect to the endogenous variables in vintage capital growth models.
} 
a continuous solution and evaluating the delayed solution values, and non-linear equation solvers for determining the meshpoints), the question of controlling the stability and numerical accuracy of the solution arises. The necessary analysis has already been performed for continuous explicit Runge-Kutta methods by Baker and Paul (1993-a, 1993-b).

\section{The Solow Vintage Capital Growth Model}

For an economic application of our algorithm, we consider a general formulation of the vintage capital growth model of Solow et al (1966). The structural equations of the model for $t \geq 0$ are:

$$
\begin{aligned}
y(t) & =\int_{t-T(t)}^{t} i(z) d z \\
l(t) & =\int_{t-T(t)}^{t} i(z) \exp \{-\gamma(z) z\} d z \\
i(t) & =s y(t)
\end{aligned}
$$

where $y(t)$ is production, $l(t)$ is labour demand, $i(t)$ is investment and is specified for $t<0$, and $T(t)$ is the age of the oldest machine still in use at time $t$. The parameter $s \in(0,1)$ and represents the saving rate.

The age structure of the technology is represented by equations (7) and (8), the production and labour demand respectively. Whilst in use, each machine of generation $t$ is assumed to require $\exp \{-\gamma(t) t\}$ workers to operate it - its labour requirement. In the following discussion, we restrict our attention to the case in which $l(t)=1$ for all $t$, that is to say the labour supply is inelastic and constant over time. Equation (9) represents the equilibrium in the goods market, under the crucial assumption that distinguishes the Solow growth model from the standard Ramsey model, namely that a fixed proportion of income is saved and invested in new machines.

The time-dependent function $\gamma(t)$ represents the technological progress. We assume that $\gamma(t) t$ is increasing with respect to time $t$. This reflects the fact that new machines are always more productive than old machines, in the sense that older machines require more workers to produce the same quantity of goods.

By differentiating the system ${ }^{9}(7)-(9)$, and after some elementary substitutions, for

\footnotetext{
${ }^{9}$ In the SVCM, the differentiability of the solution derives from a preliminary assumption on the initial investment profile, called the "no-holes" hypothesis, which states that the initial investment profile $i_{0}(t)$ is strictly positive from a given time $t^{*}<0$.
} 
$t \geq 0$ we obtain

$$
\begin{aligned}
y^{\prime}(t) & =s y(t)(1-R(t)), \\
T^{\prime}(t) & =1-\frac{i(t)}{i(t-T(t))} \times R(t), \\
i^{\prime}(t) & =s y^{\prime}(t)
\end{aligned}
$$

where

$$
R(t)=\frac{\exp \{-\gamma(t) t\}}{\exp \{-\gamma(t-T(t))(t-T(t))\}}
$$

The initial conditions $y(0), T(0)$ and $i(0)$ are obtained from $(7)-(9)$ by putting $t=0$. Since it is assumed that $\gamma(t) t$ is increasing, the production $y(t)$ and investment $i(t)$ have a trend component. Note that the rate of growth depends on the requirements ratio $R(t)$. i.e., The ratio of the labour requirement of new machines to the labour requirement of the machines that they are replacing.

As Solow et al showed, if $\gamma(t)$ is constant $(\gamma(t)=\gamma>0)$ and $s>\gamma$, then the economy converges to a balanced growth path. i.e., As $t$ goes to infinity, $T(t) \rightarrow T^{*} \equiv-\log (1-$ $\gamma / s) / \gamma$ and $y(t) \rightarrow y^{*} \exp \{\gamma t\}$, where $y^{*} \equiv 1 / s T^{*}$. An important point to note is that over the balanced growth path production is growing at the rate $\gamma$ since the requirements ratio is simply $\exp \left\{\gamma T^{*}\right\}$.

We address two numerical issues in this paper:

1. How do the initial conditions affect the short-term dynamics of the economy ${ }^{10}$ ?

2. Is the convergence result for the SVCM robust to slight modifications of the specification of the technological progress?

We use our numerical code to investigate these two issues ${ }^{\mathbf{1 1}}$.

1. Some insight into the short-term dynamics of the model ${ }^{12}$.

Figure 1 gives $T(t)$ and the detrended production $\widehat{y}(t)=y(t) \exp \{-\gamma t\}$ when the initial investment profile is exponential: $i_{0}(t)=k \exp \{\gamma t\}$ for $k>0$. In this case it is easy to show that $T(0)=1 / k$ from the structural equations. Moreover, we can also show that the investment $i(t)$ typically jumps at $t=0$, since $\lim _{t \rightarrow 0-} i(t)=k$ is generally different from

$$
i(0)=k\left(\frac{1-\exp \{-\gamma / k\}}{1-\exp \left\{-\gamma T^{*}\right\}}\right) .
$$

\footnotetext{
${ }^{10}$ As reported by Solow et al: "Since the initial capital profile is to a large extent arbitrary, we can not hope to characterize the solution (of this system) fully."

${ }^{11}$ See the Appendix for details of the numerical code. The program is written in ForTRAN 77 , and is available on request.

${ }^{12}$ We use the parameter values reported by Christiano and Eichenbaum (1992), namely $s=0.27$ and $\gamma=0.04$ so that $T^{*}=4.008$ and $y^{*}=0.924$. The corresponding period length is 2.5 years and the solution for $T^{*}$ corresponds to almost 10 years.
} 
When $k=1 / T^{*}$, the solution is trivial since $\lim _{t \rightarrow 0^{-}} i(t)=i(0)$ and the past investment $i_{0}(t)$ corresponds to the balanced growth path. When $k<1 / T^{*}$ (resp. $k>1 / T^{*}$ ), the economy starts with a low (resp. high) stock of machines relative to the balanced growth path. We can see from Figure 1 that the initial lifetime of capital must be greater (resp. smaller) than $T^{*}$ to allow the labour market to clear $^{13}$. After a jump in the investment at $t=0$, since $\lim _{t \rightarrow 0-} i(t)$ is smaller (resp. greater) than $i(0)$, the model converges monotonically to the balanced growth path.

\section{Robustness of convergence properties with respect to technological progress.}

To investigate this question, we consider a time-dependent technological progress with a periodic component that vanishes asymptotically, so that we recover the Solow et al specification of the technological progress at the limit. We specify the technological progress as follows:

$$
\gamma(t)=\gamma+\alpha \frac{\sin (\omega t)}{t} \text { where } \alpha>0 \text { and } \omega>0
$$

with $\gamma>\alpha \omega$ to ensure that $\gamma(t) t$ is an increasing function. The ratio $\alpha / t$ determines the size of the maximum fluctuation in the technological progress with $t$. In our simulations, we chose a small value for $\alpha$, and consequently the periodic component of the technological progress vanishes quickly. However, the periodic component of the requirements ratio does not necessarily vanish as $t$ increases, since the requirements ratio is

$$
\exp \{\gamma T(t)+\alpha[\sin (\omega(t-T(t)))-\sin (\omega t)]\}
$$

Despite the fact that our specification of the technological progress ensures that it converges quickly to the SVCM, we obtain two different types of long-term dynamics depending on the periodicity parameter ${ }^{14} \omega$. In Figures $2 \mathrm{a}$ and $2 \mathrm{~b}$, we took $i_{0}(t)=k \exp \{\gamma(t) t\}$ for $t<0$ with $k=1 / T^{*}$. In Figure $2 \mathrm{a}$, we set the parameter $\omega=2 \pi / T^{*}$, so that $\sin (\omega t)$ has periodicity $T^{*}$. We can see that after an initial adjustment period of length $T^{*}$, both $T(t)$ and $\hat{y}(t)$ are very close to the long-term solution values $T^{*}$ and $y^{*}$, respectively. The main economic reason for this is that the periodic component of the requirements ratio vanishes after a few periods. However, as we can see in Figure 2b, when the periodicity is not a multiple of $T^{*}$ (here $\omega=\pi / 5$, so that the periodicity is 10 ), the periodic component of the requirements ratio never vanishes and so both $T(t)$ and $\widehat{y}(t)$ are periodic with the same periodicity of $\sin (\omega t)$.

In order to show that these findings are robust with respect to the specification of the initial investment profile, we ran the same simulations again with $\omega=2 \pi / T^{*}$ and $\omega=\pi / 5$,

\footnotetext{
${ }^{13}$ These arguments are independent of the specification of the initial investment profile.

${ }^{14}$ For the numerical simulations, we used the values $\alpha=0.02, s=0.27$ and $\gamma=0.04$.
} 
but with the original exponential initial investment profile, $i_{0}(t)=k \exp \{\gamma t\}$. Figures $3 \mathrm{a}$ and $3 \mathrm{~b}$ give the corresponding solutions, respectively.

\section{Conclusion: A Crucial Remaining Issue}

In this paper, we examined techniques for the solution of state-dependent DDEs, using simple examples to illustrate the arguments. We also provided an economic application as an example of the useful insight that can be gained from numerical simulations. However, this paper is designed more to stimulate interest in the field of computational economics rather than to provide definitive statements about vintage capital growth models, and in particular the SVCM.

A major issue still to be resolved is the numerical solution of so-called mixed-DDEs, namely DDEs with both endogenous lags and leads. The numerical techniques presented in this paper only permit models with lags to be solved. However they can be easily adapted to solve equations that only have leads, so long as the "final solution" is specified. The solution is computed by making the substitution $t \rightarrow-s$ to obtain a normal DDE. This idea was suggested, for example, by Bellman and Cooke (1963), Chapter 3, for fixedleads. However, these solution techniques cannot be applied to mixed-DDEs. But, as stated by Boucekkine et al (1995), such mixed-delay systems do occur in the general formulation of the Ramsey vintage capital growth model. In the SVCM, since investment is proportional to production, the replacement decisions are only dependent on previously calculated quantities. Although the general vintage capital growth model should include a backward-looking component (representing the history of capital accumulation within the economy), it should also include a forward-looking component (representing investment decisions which are dependent on future profits, and, in particular, on the life time of these machines).

A mixed-delay model was recently analyzed by Caballero and Hammour (1994), under the assumption that the solutions are periodic ${ }^{15}$. Having assumed that the solutions are periodic, they use a multiple-shooting technique to compute the one-period solution path and then use a predictor-corrector scheme to extend the solution to the whole real-time interval. Although Caballero and Hammour fail to justify their periodicity assumption and do not rigorously establish the convergence of their numerical method, their simulations are certainly worthwhile as they highlight the extreme difficulty of solving mixed-DDEs. However, their periodicity assumption does mean that their approach cannot be used to

\footnotetext{
${ }^{15}$ This assumption is implicitly "justified" by the fact that the differential-difference model has a forcing (exogenous) periodic function, namely the aggregate demand.
} 
solve general mixed-DDEs. In fact, there exists very little in the mathematical literature on the numerical solution of mixed-DDEs ${ }^{16}$.

This situation is the same as the one faced by economists at the beginning of the eighties for the numerical solution of non-linear rational expectation models with both lags and leads. Their numerical approach consists of simultaneously solving the modelling equations on a fixed time interval. Unfortunately, the solution technique discussed in this paper cannot be applied in the same manner. One strategy that appears to be feasible is to combine the numerical techniques mentioned in this paper with a predictor-corrector strategy. However, this task is far from trivial, and we expect it to stimulate further research and debate.

\footnotetext{
${ }^{16} \mathrm{Most}$ probably because mixed-DDEs do not occur in the natural world, which motivates most computational mathematics.
} 


\section{References}

Baker, C.T.H. and C.A.H. Paul, 1993-a, Parallel continuous Runge-Kutta methods and vanishing lag delay differential equations, Advances in Computational Mathematics 1, 367394.

Baker, C.T.H. and C.A.H. Paul, 1993-b, A global convergence theorem for a class of parallel continuous explicit Runge-Kutta methods and vanishing lag delay differential equations, SIAM Journal of Numerical Analysis, forthcoming.

Bellman, R.E. and K.L. Cooke, 1963, Differential-difference equations, Mathematics in Science and Engineering 6 (Academic Press, London).

Benhabib, J. and A. Rustichini, 1991, Vintage capital, investment and growth, Journal of Economic Theory 55, 323-339.

Boucekkine, R., M. Germain and O. Licandro, 1995, Creative destruction and business cycles, Universidad Carlos III de Madrid WP 95-17.

Caballero, R.J. and M.L. Hammour, 1994, The cleansing effects of recessions, American Economic Review 84, 1350-1368.

Christiano, L.J. and M. Eichenbaum, 1992, Current real business theories and aggregate labor market fluctuations, American Economic Review 82, 430-450.

Cooley, T.F., J. Greenwood and M. Yorukoglu, 1994, The replacement problem, Institute for Empirical Macroeconomics DP No. 95.

Dormand, J.R. and P.J. Prince, 1980, A family of embedded Runge-Kutta formulae, Journal of Computational and Applied Mathematics 6, 19-26.

Malcomson, J., 1975, Replacement and the rental value of capital equipment subject to obsolescence, Journal of Economic Theory 10, 24-41.

Shampine, L.F., 1985, Interpolation for Runge-Kutta methods, SIAM Journal of Numerical Analysis 22, 1014-1026.

Solow, R., J. Tobin, C. Von Weizsacker and M Yaari, 1966, Neoclassical growth with fixed factor proportions, Review of Economic Studies 33, 79-115.

Van Hilten, O., 1991, The optimal lifetime of capital equipment, Journal of Economic Theory 55, 449-454.

Willé, D.R. and C.T.H. Baker, 1992, The tracking of derivative discontinuities in systems of delay differential equations, Applied Numerical Mathematics 9, 223-234. 


\section{Appendix: The Numerical Code}

The numerical code used to solve the DDEs in this paper is based on the fifth-order explicit Runge-Kutta method of Dormand and Prince (1980). It uses the fifth-degree fifth-order Hermite interpolant due to Shampine (1985) to evaluate delayed solution values, and the predictor-corrector iteration of Baker and Paul (1993-a, 1993-b) to solve vanishing lag DDEs. In addition, it allows the user to track the propagation of derivative discontinuities in the solution (see Wille and Baker (1992)) to improve the efficiency and reliability of the code. When the position of a derivative discontinuity depends on one or more solution values from the current interval, it extrapolates the solution from the last accepted step in order to estimate the position of the discontinuity, and improves this estimate as the solution is advanced. 
FIGURE 1: Solow model with $i_{0}(t)=k \exp \{\gamma t\}$.

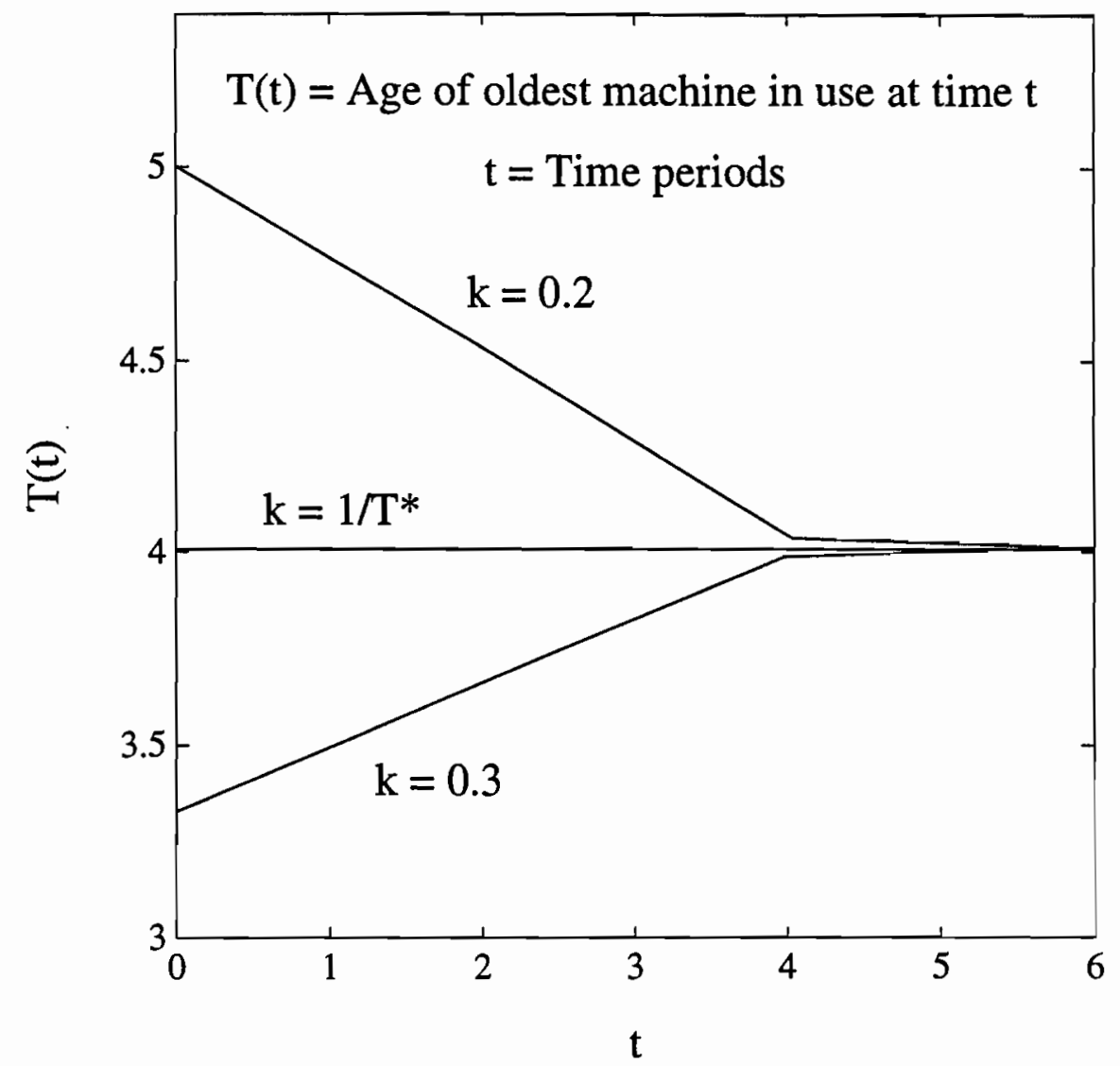


FIGURE 1(continuation): Solow model with $i_{0}(t)=k \exp \{\gamma t\}$.

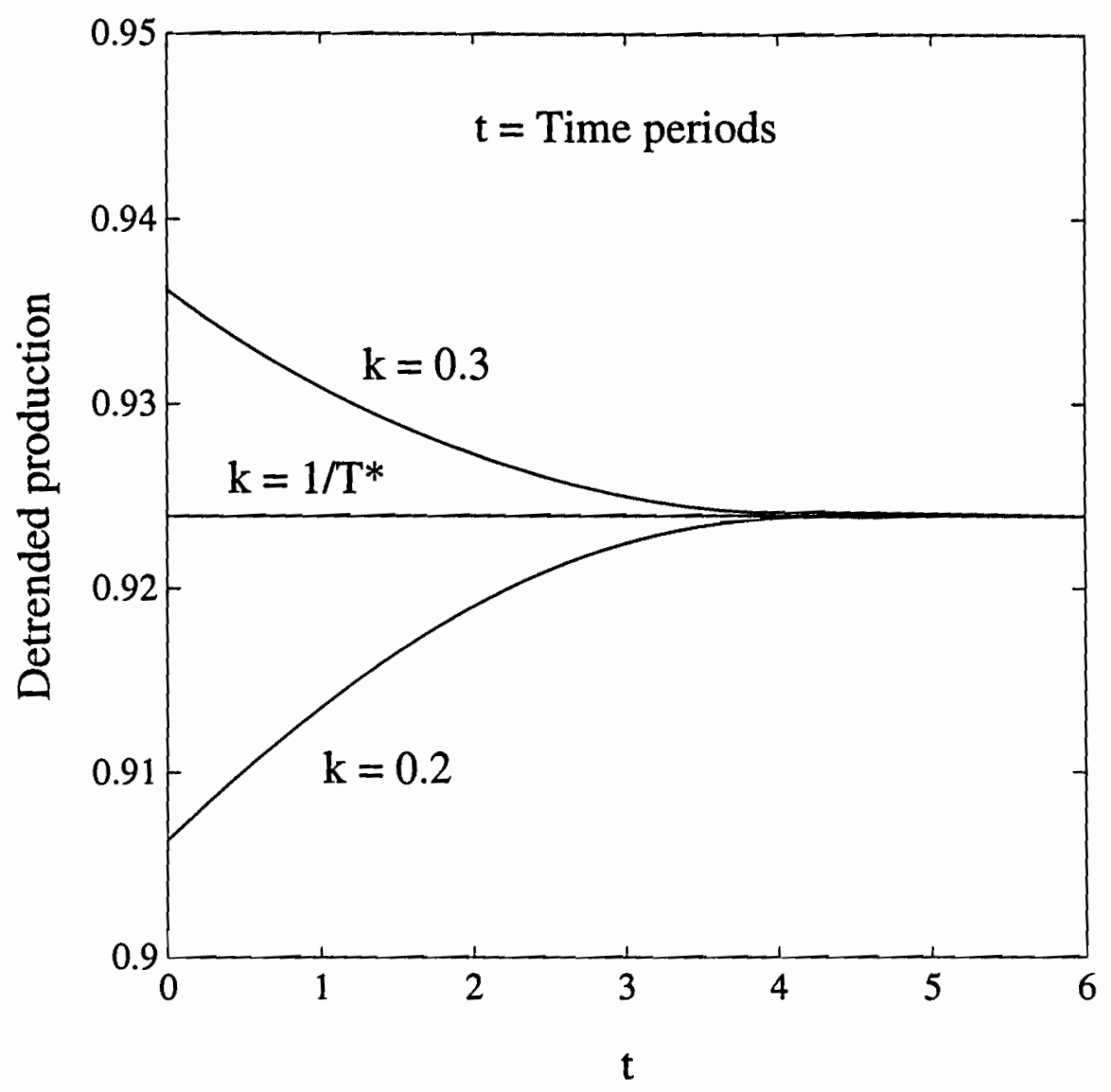


FIGURE 2A: Extended Solow model with $i_{0}(t)=k \exp \{\gamma(t) t\}$ and $\omega=2 \pi / T^{*}$.

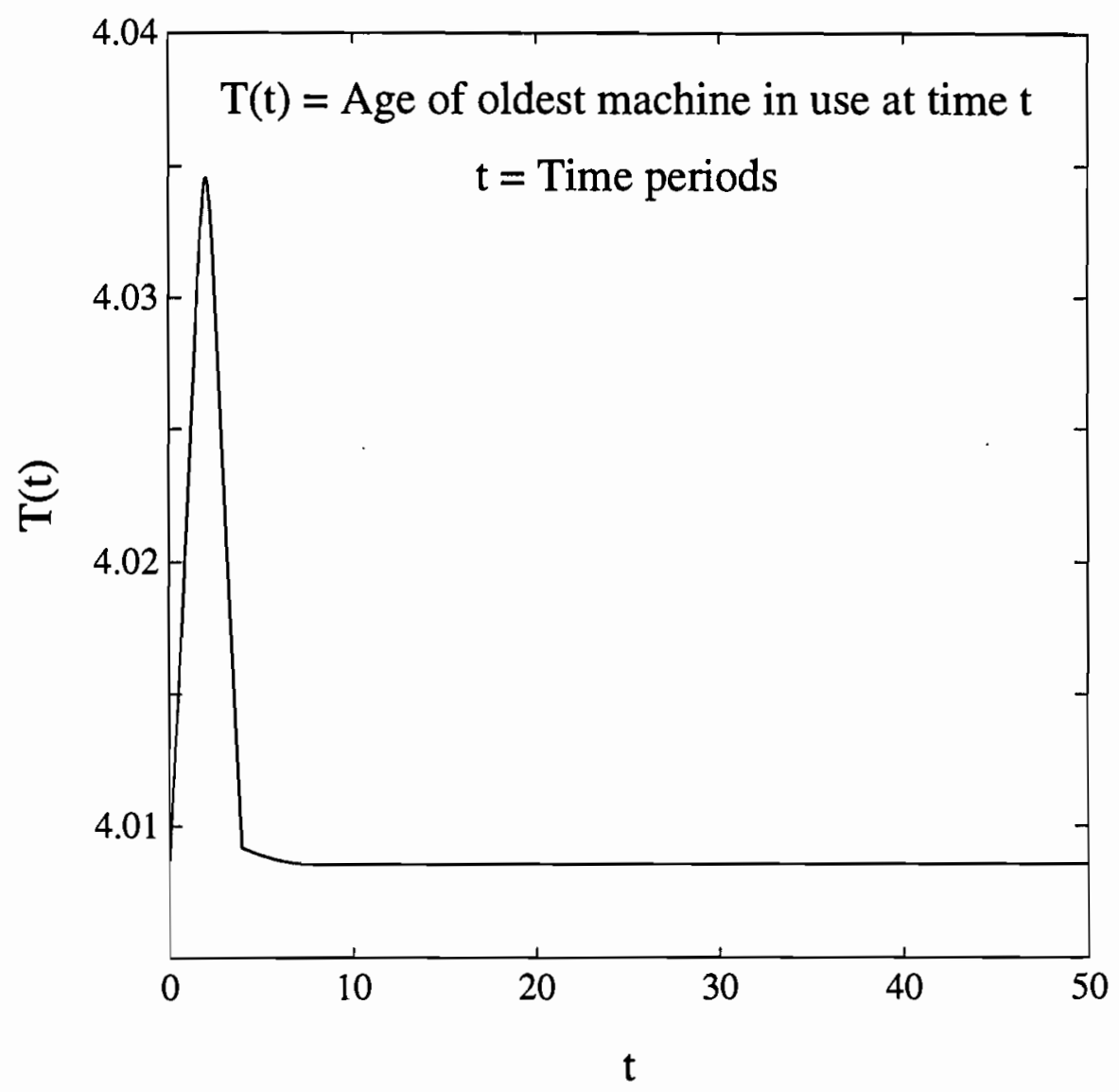


FIGURE 2A(continuation): Extended Solow model with $i_{0}(t)=k \exp \{\gamma(t) t\}$ and $\omega=2 \pi / T^{*}$.

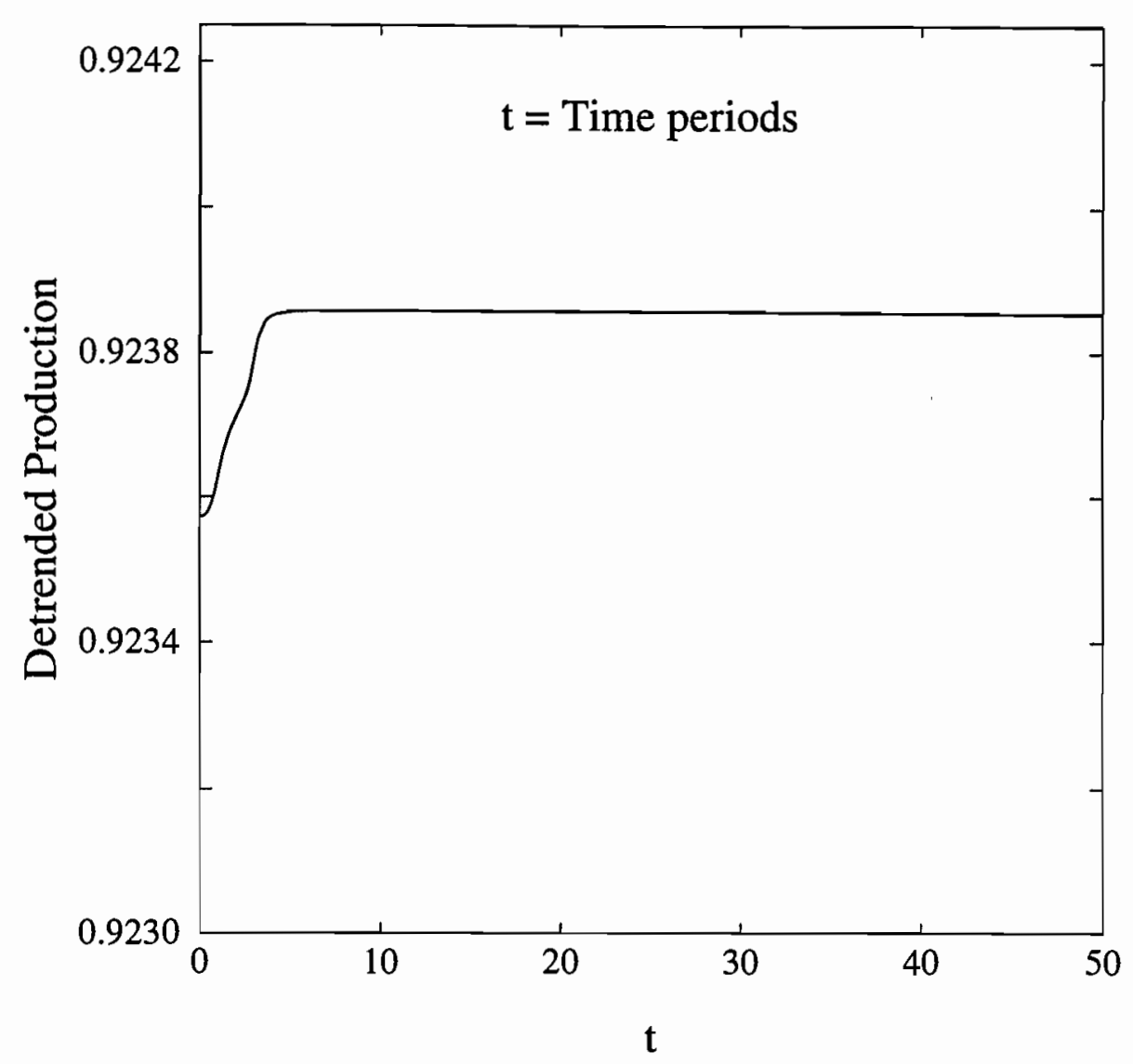


FIGURE 2B: Extended Solow model with $i_{0}(t)=k \exp \{\gamma(t) t\}$ and $\omega=\pi / 5$.

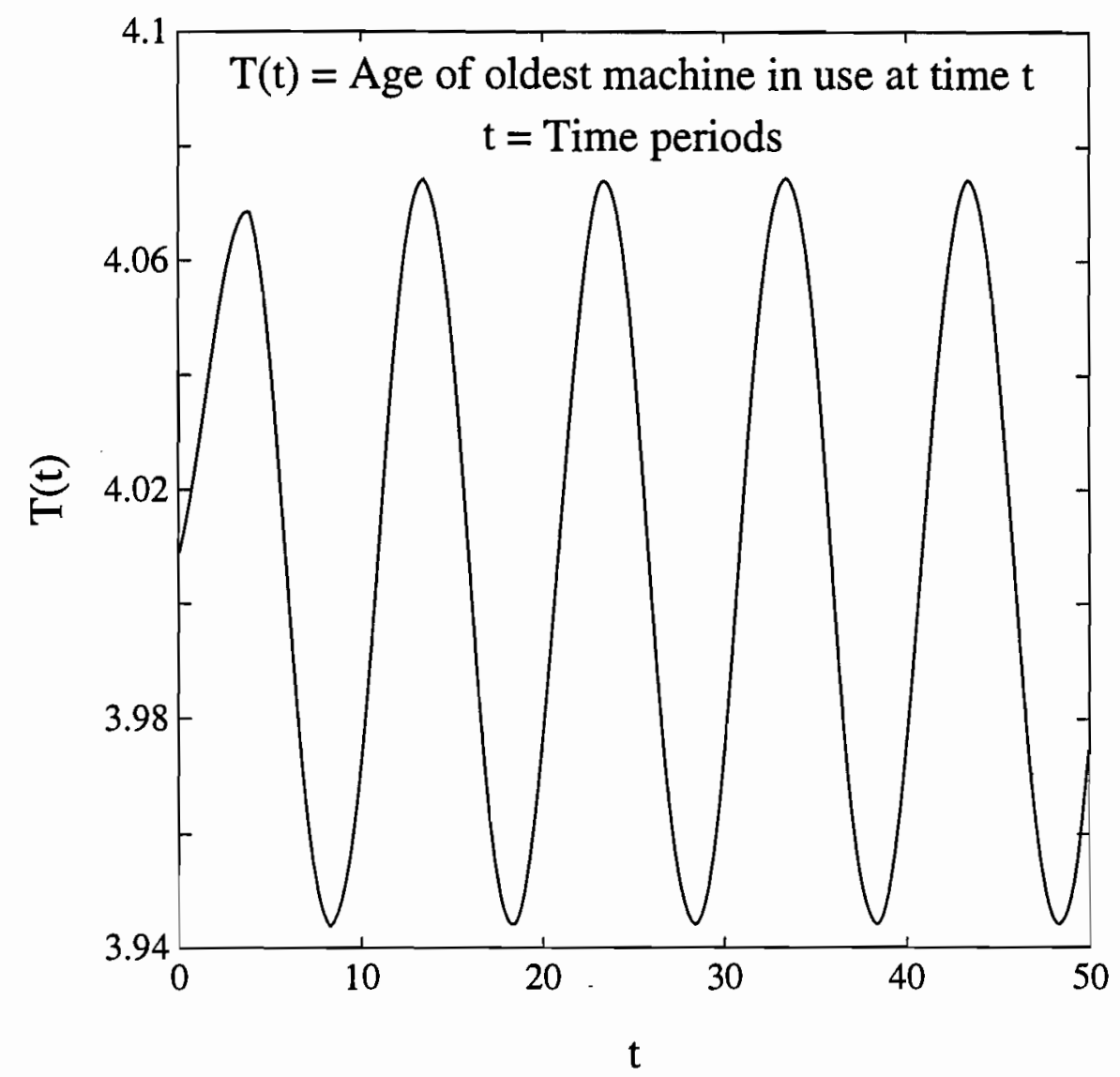


FIGURE 2B(continuation): Extended Solow model with $i_{0}(t)=k \exp \{\gamma(t) t\}$ and $\omega=\pi / 5$.

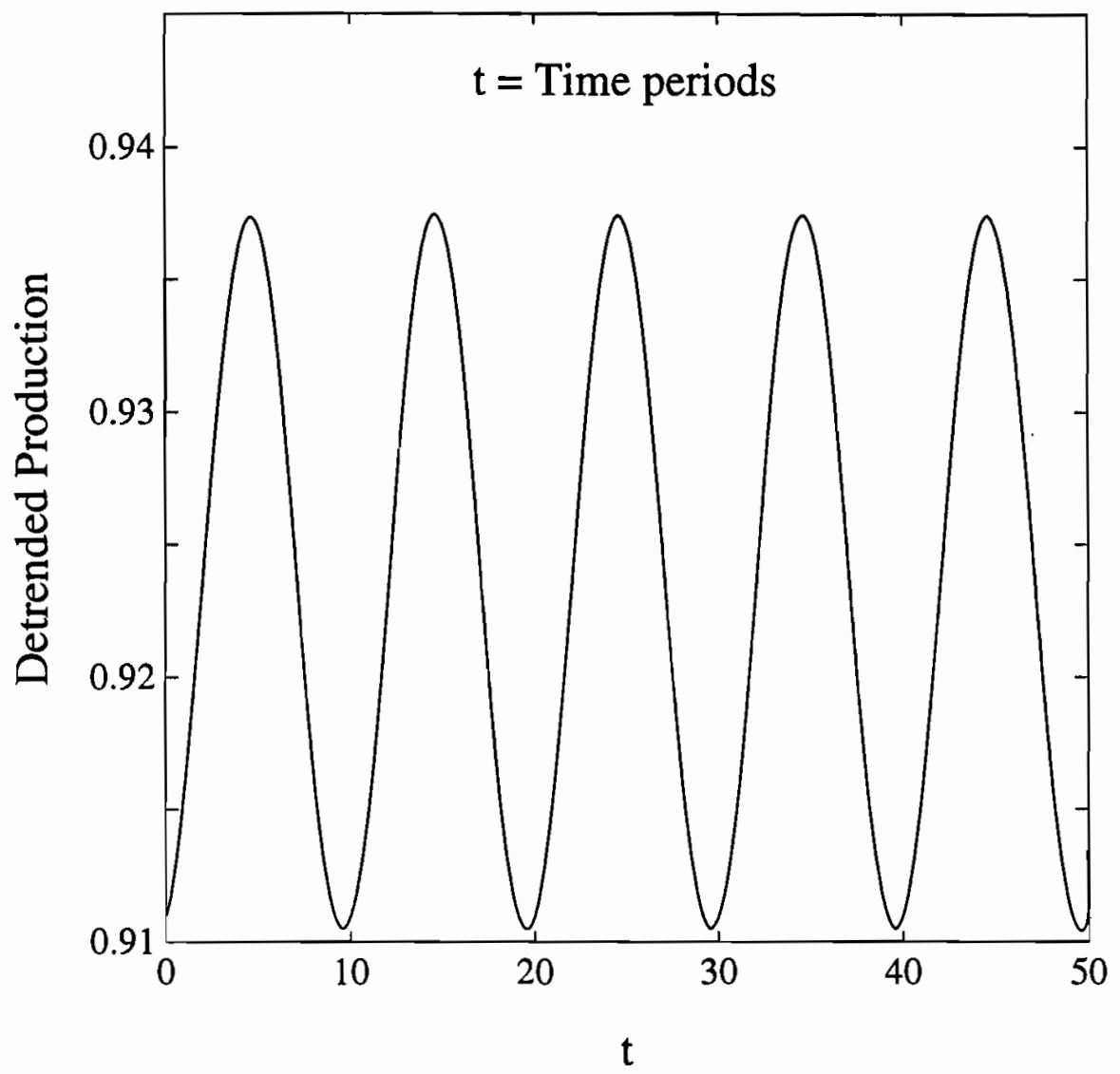


FIGURE 3A: Extended Solow model with $i_{0}(t)=k \exp \{\gamma t\}$ and $\omega=2 \pi / T^{*}$.

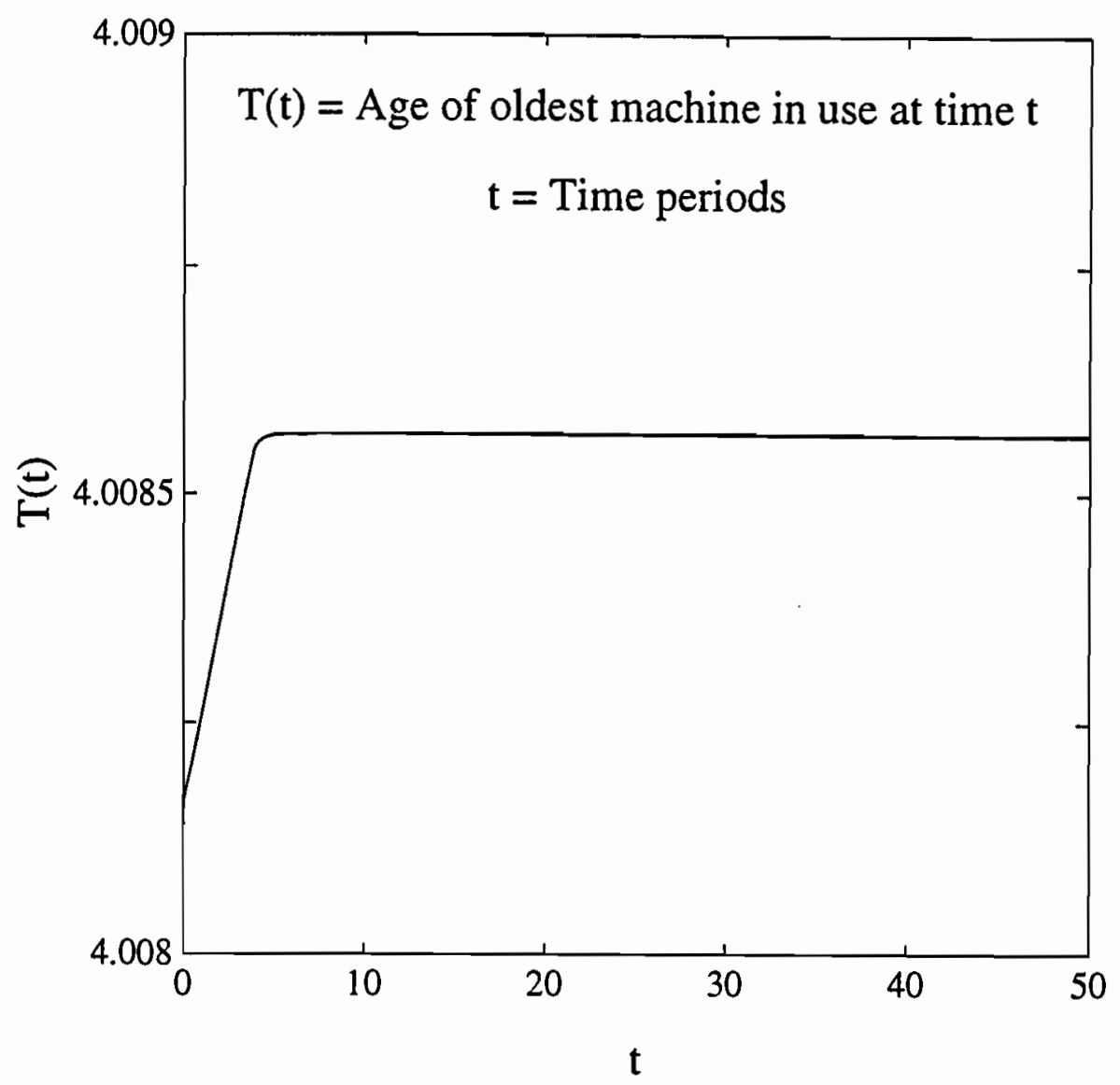


FIgURE 3A(continuation): Extended Solow model with $i_{0}(t)=k \exp \{\gamma t\}$ and $\omega=2 \pi / T^{*}$.

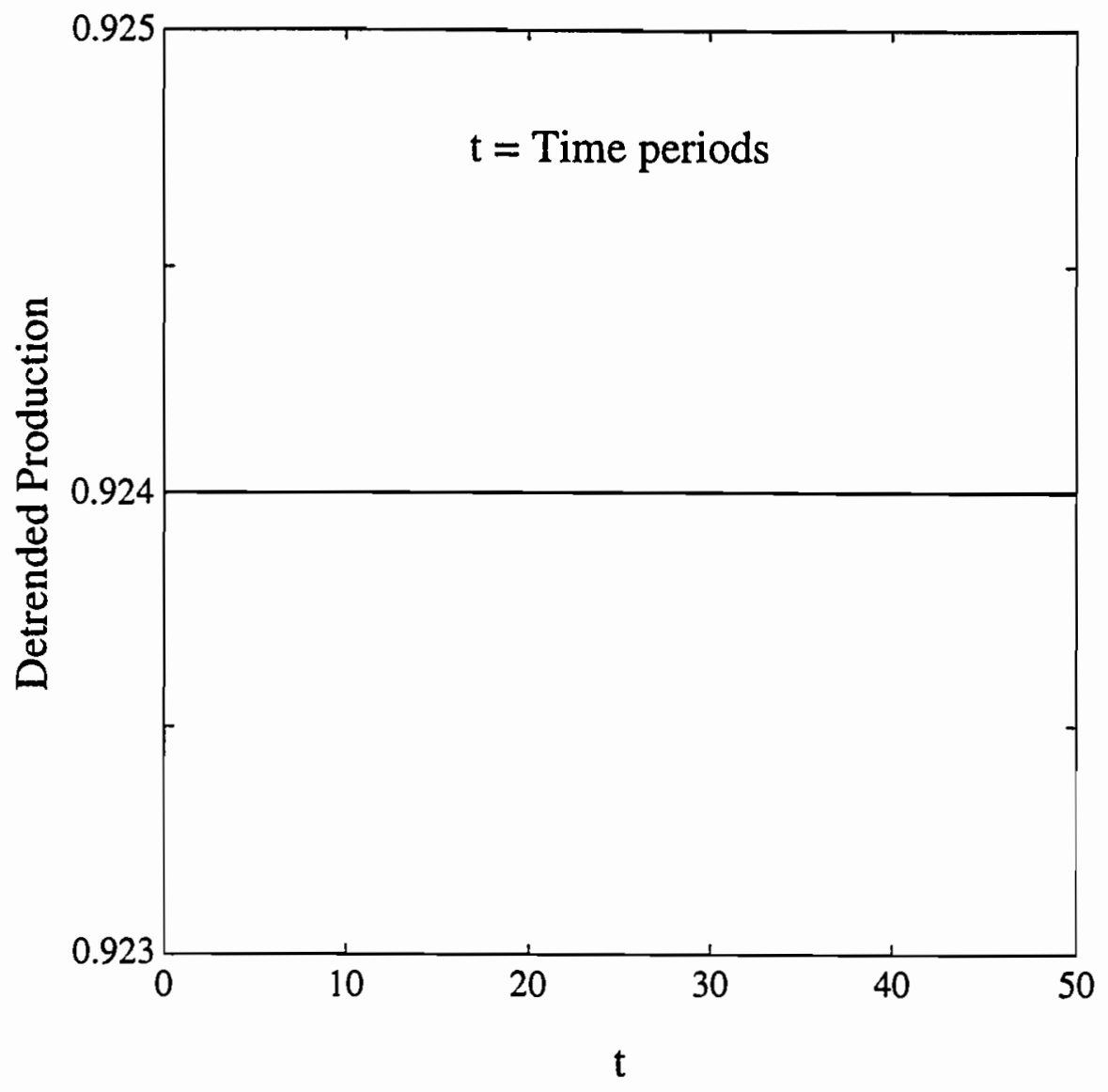


Figure 3B: Extended Solow model with $i_{0}(t)=k \exp \{\gamma t\}$ and $\omega=\pi / 5$.

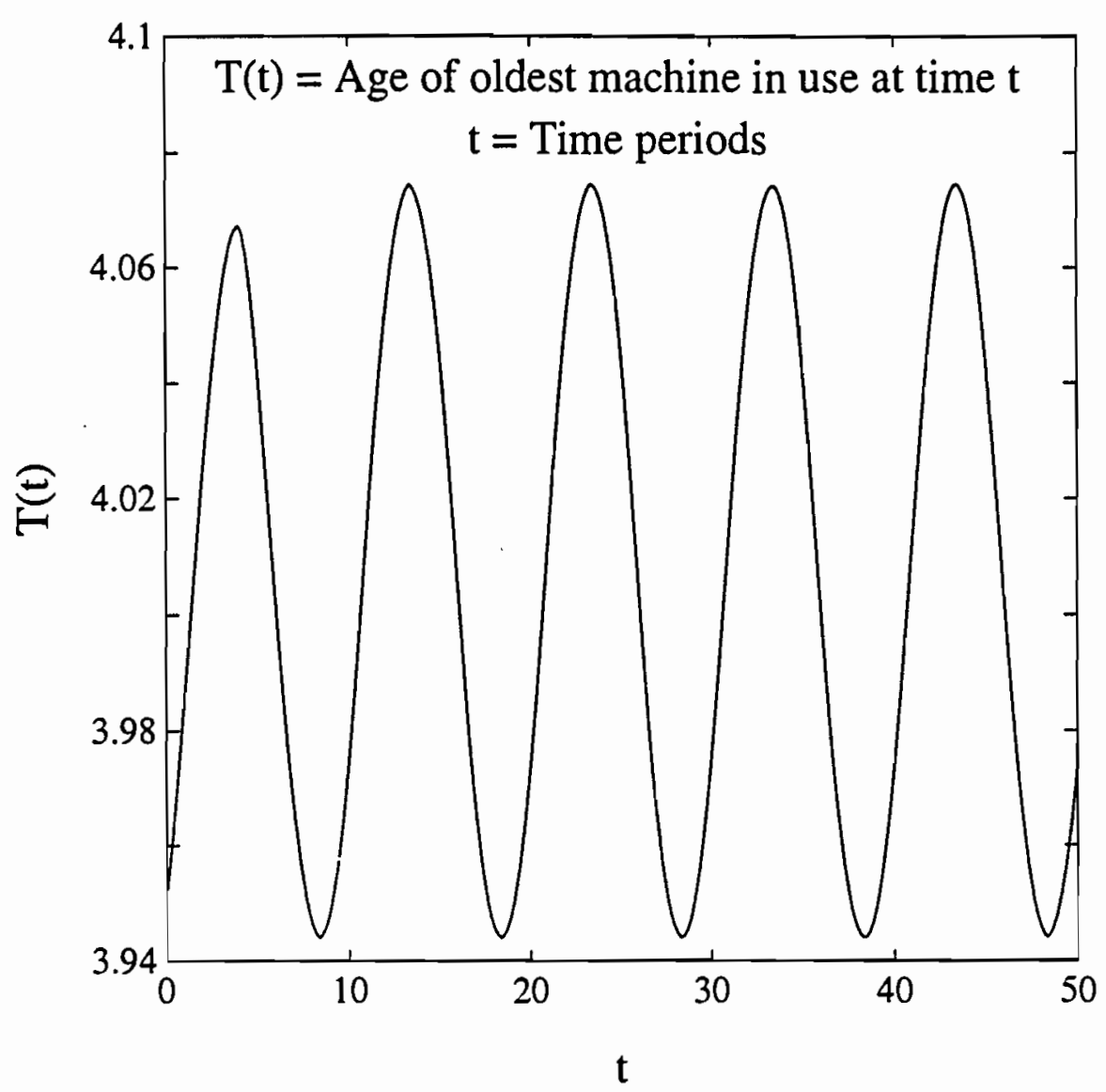


FIgURE 3B(continuation): Extended Solow model with $i_{0}(t)=k \exp \{\gamma t\}$ and $\omega=\pi / 5$.

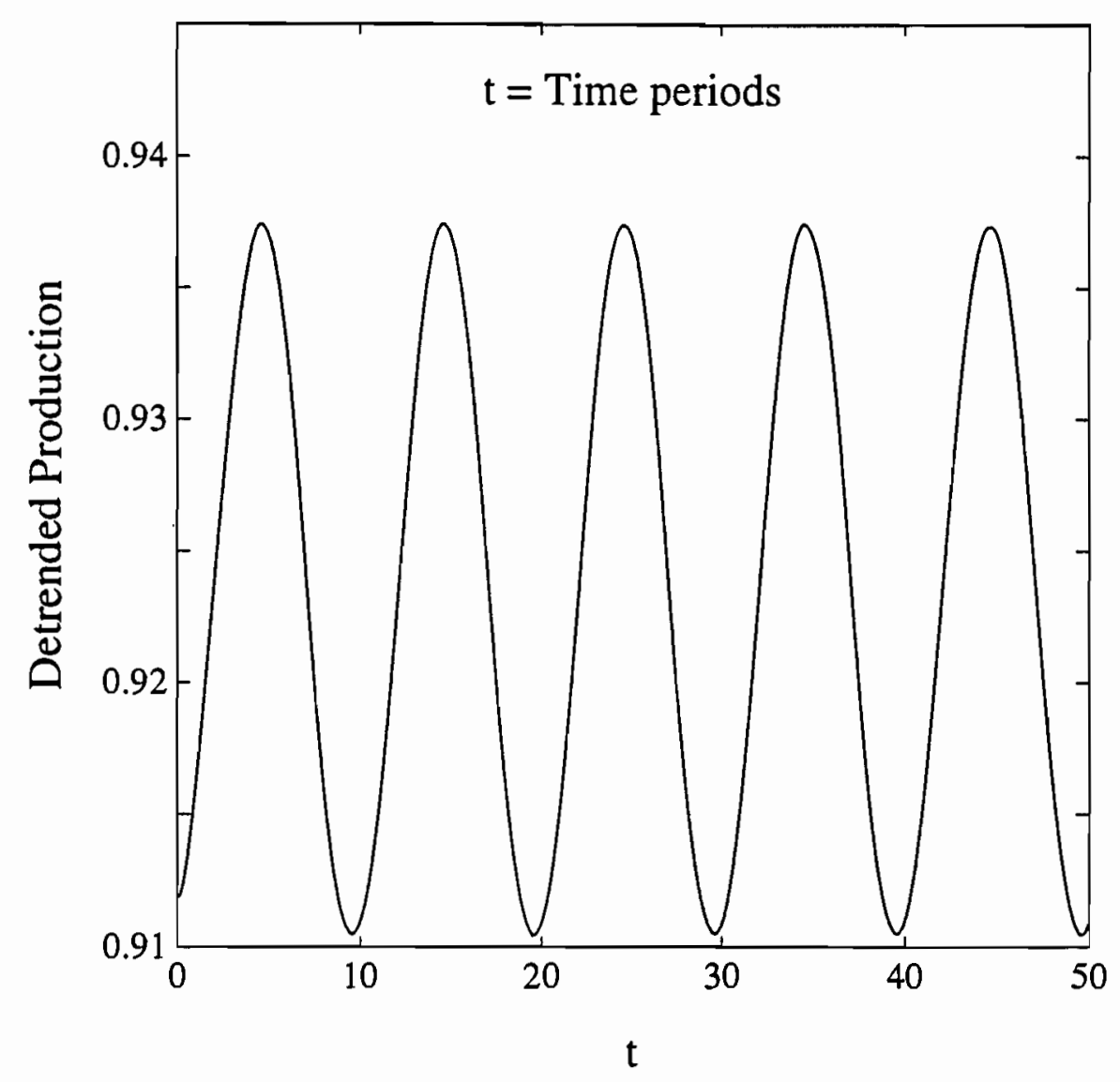

